\title{
Halo Structure of ${ }^{14} \mathrm{Be}$
}

M. Labiche, ${ }^{1, *}$ N. A. Orr,${ }^{1, \dagger}$ F. M. Marqués, ${ }^{1}$ J. C. Angélique,${ }^{1}$ L. Axelsson, ${ }^{2}$ B. Benoit,${ }^{3}$ U. C. Bergmann, ${ }^{4}$ M. J. G. Borge, ${ }^{5}$ W. N. Catford, ${ }^{6}$ S. P. G. Chappell, ${ }^{7}$ N. M. Clarke,${ }^{8}$ G. Costa,,${ }^{9}$ N. Curtis,,${ }^{6,8}$ A. D' Arrigo, ${ }^{3}$ E. de Góes Brennand ${ }^{3}$ O. Dorvaux,${ }^{9}$ G. Fazio, ${ }^{10}$ M. Freer,${ }^{1,8}$ B. R. Fulton, ${ }^{8}$ G. Giardina, ${ }^{10}$ S. Grévy, ${ }^{11,1}$ D. Guillemaud-Mueller, ${ }^{11}$ F. Hanappe, ${ }^{3}$ B. Heusch, ${ }^{9}$ K. L. Jones, ${ }^{6}$ B. Jonson, ${ }^{2}$ C. Le Brun, ${ }^{1}$ S. Leenhardt, ${ }^{11}$ M. Lewitowicz, ${ }^{12}$ M. J. Lopez, ${ }^{12}$ K. Markenroth, ${ }^{2}$ A. C. Mueller, ${ }^{11}$ T. Nilsson, ${ }^{2,}$ A. Ninane, ${ }^{1, \S}$ G. Nyman, ${ }^{2}$ F. de Oliveira, ${ }^{12}$ I. Piqueras, ${ }^{5}$ K. Riisager, ${ }^{4}$ M. G. Saint Laurent, ${ }^{12}$ F. Sarazin,,${ }^{12, \|}$ S. M. Singer, ${ }^{8}$ O. Sorlin, ${ }^{11}$ and L. Stuttgé ${ }^{9}$

${ }^{1}$ Laboratoire de Physique Corpusculaire, ISMRA et Université de Caen, IN2P3-CNRS, F-14050 Caen Cedex, France

${ }^{2}$ Experimentall Fysik, Chalmers Tekniska Högskola, S-412 96 Göteborg, Sweden

${ }^{3}$ Université Libre de Bruxelles, PNTPM, CP 226, B-1050 Bruxelles, Belgium

${ }^{4}$ Institut for Fysik og Astronomi, Aarhus Universitet, DK-8000 Aarhus C, Denmark

${ }^{5}$ Instituto de Estructura de la Materia, CSIC, E-28006 Madrid, Spain

${ }^{6}$ Department of Physics, University of Surrey, Guildford, GU2 7XH, United Kingdom

${ }^{7}$ Nuclear and Astrophysics Laboratory, University of Oxford, Oxford OX1 3RH, United Kingdom

${ }^{8}$ School of Physics and Astronomy, University of Birmingham, Birmingham B15 2TT, United Kingdom

${ }^{9}$ Institut de Recherches Subatomique, IN2P3-CNRS, Université Louis Pasteur, F-67037 Strasbourg Cedex, France

${ }^{10}$ Dipartimento di Fisica, Università di Messina, I-98166 Messina, Italy

${ }^{11}$ Institut de Physique Nucléaire, IN2P3-CNRS, F-91406 Orsay Cedex, France

${ }^{12}$ GANIL (CEA/DSM-CNRS/IN2P3), BP 55027, F-14076 Caen Cedex, France

(Received 6 June 2000)

\begin{abstract}
The two-neutron halo nucleus ${ }^{14} \mathrm{Be}$ has been investigated in a kinematically complete measurement of the fragments $\left({ }^{12} \mathrm{Be}\right.$ and neutrons) produced in dissociation at $35 \mathrm{MeV} /$ nucleon on $\mathrm{C}$ and $\mathrm{Pb}$ targets. Two-neutron removal cross sections, neutron angular distributions, and invariant mass spectra were measured, and the contributions from electromagnetic dissociation (EMD) were deduced. Comparison with three-body model calculations suggests that the halo wave function contains a large $\nu\left(2 s_{1 / 2}\right)^{2}$ admixture. The EMD invariant mass spectrum exhibited enhanced strength near threshold consistent with a nonresonant soft-dipole excitation.
\end{abstract}

DOI: 10.1103/PhysRevLett.86.600

The size and distribution of matter in the nucleus have long played a central role in nuclear physics. In stable nuclei the neutron and proton distributions exhibit essentially identical radii. In contrast, for some light nuclei far from stability, which combine a large neutron excess with very weak binding, large differences have been found. Such "halo" systems are well described by a core, resembling a normal nucleus, surrounded by an extended valence neutron density distribution [1].

In general terms the halo may be regarded as a threshold phenomenon whereby the loosely bound valence neutrons tunnel with significant probability into the region outside the core potential. Within a simple quasideuteron description, the extent of the halo is governed by the separation energy and reduced mass of the system [2]. Under more realistic considerations the development of the halo is also influenced by the centrifugal barrier [3]. In the cases of ${ }^{6,8} \mathrm{He},{ }^{11} \mathrm{Be}$, and ${ }^{11} \mathrm{Li}$, which have been investigated experimentally in considerable detail, the valence neutrons occupy the $2 s_{1 / 2}$ and/or $1 p_{3 / 2,1 / 2}$ single-particle orbitals. In ${ }^{14} \mathrm{Be}$ the configuration of the halo neutrons would, in a naive shell model prescription, be $\nu\left(d_{5 / 2}\right)^{2}$. More sophisticated models suggest, however, that a $\nu\left(s_{1 / 2}\right)^{2}$ admixture is also present [4-7]. Unfortunately, a paucity of experimental data $[8-10]$ has precluded the elucidation of the struc-
PACS numbers: 27.20. +n, 24.30.Gd, 25.60.Dz, 25.60.Gc

ture of ${ }^{14} \mathrm{Be}$ beyond the matter radius [11-14]. Compared to the other halo systems, the comparatively strong binding of the valence neutrons in ${ }^{14} \mathrm{Be}\left(S_{2 n}=1.34 \pm 0.11 \mathrm{MeV}\right.$ $[15,16])$ combined with the $\nu\left(d_{5 / 2}\right)^{2}$ component may provide a new window on continuum excitations, including the long sought after soft-dipole resonance $[17,18]$.

The goal of the present study was thus to explore the halo structure and continuum excitations of the twoneutron halo nucleus ${ }^{14} \mathrm{Be}$. The tool chosen was a kinematically complete measurement of the fragments $\left({ }^{12} \mathrm{Be}\right.$ and two neutrons) from the dissociation of a ${ }^{14} \mathrm{Be}$ beam on $\mathrm{C}$ and $\mathrm{Pb}$ targets. Such a measurement allowed the twoneutron removal cross sections, neutron angular distributions, and invariant mass spectra to be extracted (an analysis of the neutron-neutron correlations has been presented elsewhere [19]). The use of $\mathrm{C}$ and $\mathrm{Pb}$ targets permitted the electromagnetic component of the dissociation (EMD) to be deduced.

The ${ }^{14} \mathrm{Be}$ beam $(\bar{E}=35 \mathrm{MeV} /$ nucleon, $\sim 130 \mathrm{pps})$ was prepared from a $63 \mathrm{MeV} /$ nucleon ${ }^{18} \mathrm{O}$ primary beam using the LISE3 spectrometer at GANIL. The energy spread in the beam was $10 \%$ and was compensated for by a time-of-flight measurement over a $24 \mathrm{~m}$ flight path between a parallel-plate avalanche counter (PPAC) located at the first focus of the spectrometer and the beam 
identification $\mathrm{Si}$ detector $(300 \mu \mathrm{m})$ located just upstream of the targets. The beam particles were tracked onto the breakup targets (C $275 \mathrm{mg} / \mathrm{cm}^{2}, \mathrm{~Pb}$ $570 \mathrm{mg} / \mathrm{cm}^{2}$ ) using two position sensitive PPAC's (resolution $\mathrm{FWHM} \approx 1-2 \mathrm{~mm}$ ). The charged fragments from breakup were identified using a large area $\left(5 \times 5 \mathrm{~cm}^{2}\right)$ position sensitive $(\mathrm{FWHM} \approx 0.5 \mathrm{~mm}) \mathrm{Si}-\mathrm{CsI}$ telescope ( $\mathrm{Si}$ $500 \mu \mathrm{m}$, CsI $2.5 \mathrm{~cm}$ ) centered at zero degrees and located $11.4 \mathrm{~cm}$ downstream of the target. The energy response of the telescope $(\mathrm{FWHM}=1.5 \%$ ) was calibrated using various mixed secondary beams containing ${ }^{12} \mathrm{Be}$ with energies straddling that expected for ${ }^{12} \mathrm{Be}$ fragments from the dissociation of ${ }^{14} \mathrm{Be}$. In order to account for events arising from reactions in the telescope, data were also acquired without a reaction target and the beam energy reduced by an amount corresponding to the energy loss in the target.

The neutrons emitted at forward angles were detected using the 99 elements of the DEMON array [20]. The array covered angles between $+13^{\circ}$ and $-40^{\circ}$ in the horizontal plane and $\pm 14^{\circ}$ in the vertical with the modules arranged in a staggered configuration at distances between 2.5 and $6.5 \mathrm{~m}$ from the target [20]. Such a geometry provided for a relatively high two-neutron detection efficiency $(1.5 \%)$ while reducing the rate of cross talk - both intrinsically and via the use of an off-line rejection algorithm - to negligible levels [20,21]. A threshold of $15 \mathrm{MeV}$ on the neutron energy was applied in the off-line analysis to eliminate the small number of evaporation neutrons arising from the target [21].

The results obtained for the two-neutron removal cross sections, $\sigma_{-2 n}$ ( ${ }^{12} \mathrm{Be}$ identified in the telescope), the single-neutron angular distributions, $d \sigma / d \Omega\left({ }^{12} \mathrm{Be}\right.$ and neutron), and the associated angle integrated $\left(0^{\circ}-40^{\circ}\right)$ cross sections, $\sigma_{n}$, are displayed in Table I and Fig. 1a. In addition, the average neutron multiplicities have been derived $\left(\bar{m}_{n}=\sigma_{n} / \sigma_{-2 n}\right)$ and are also listed. The singleneutron angular distributions were well characterized by a Lorentzian line shape [8,21] and the corresponding momentum width parameters, $\Gamma_{n}$, have been tabulated.

In terms of the mechanisms leading to dissociation, unless the halo neutrons are highly spatially correlated, nuclear breakup will proceed via single-neutron removal (absorption or diffraction) followed by the in-flight decay of ${ }^{13} \mathrm{Be}$ [22]. This process is reflected in the narrow single-neutron angular distribution for the $\mathrm{C}$ target which is well reproduced assuming passage via a low-lying resonance in ${ }^{13} \mathrm{Be}$ [21,23]. Furthermore, as approximately equal contributions are expected for absorption and diffrac-

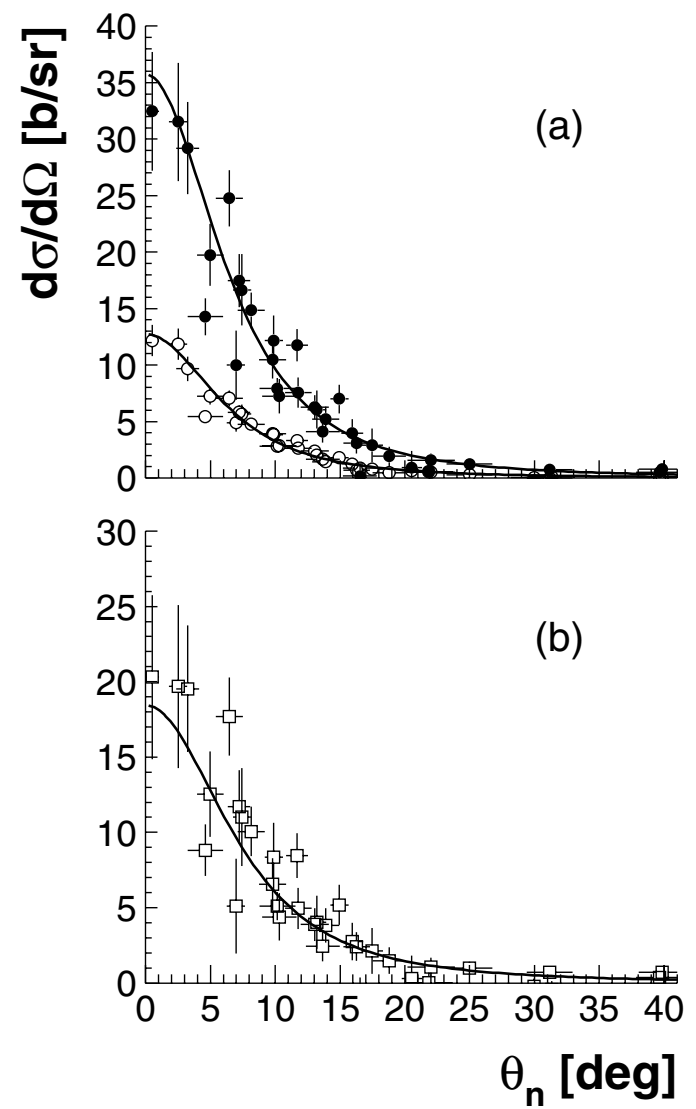

FIG. 1. (a) Single-neutron angular distributions for dissociation on $\mathrm{C}$ (open symbols) and $\mathrm{Pb}$ (solid points). The results for $\mathrm{C}$ have been scaled by a factor of 1.8 so as to represent the nuclear contribution to dissociation on $\mathrm{Pb}$ (see text). (b) Deduced EMD single-neutron angular distribution for reactions on $\mathrm{Pb}$.

tion [22] the average neutron multiplicity should be 1.5 , in accordance with that measured here.

Turning to the $\mathrm{Pb}$ target, where nuclear and Coulomb breakup is present, the enhanced cross section is indicative of a substantial EMD contribution. Assuming that the nuclear-Coulomb interference is small, the $\mathrm{C}$ target data (which arises essentially from nuclear induced reactions) may be scaled to estimate the nuclear contribution to breakup on $\mathrm{Pb}[18,21]$. Given a root-mean-square radius of $3.2 \mathrm{fm}$ for ${ }^{14} \mathrm{Be}[13,14], \sigma_{-2 n}^{\text {nucl }}(\mathrm{Pb})=0.85 \pm 0.07 \mathrm{~b}$ and, consequently, $\sigma_{-2 n}^{\mathrm{EMD}}(\mathrm{Pb})=1.45 \pm 0.40 \mathrm{~b}$. The latter can be compared to the value of $0.47 \pm 0.15 \mathrm{~b}$ measured at $800 \mathrm{MeV} /$ nucleon [18]. Importantly, for halo nuclei, the EMD cross section is dominated by the $E 1$ component [24]. An enhancement with decreasing beam energy is thus expected, owing to the large amount of dipole strength

TABLE I. Measured cross sections, average neutron multiplicities, and neutron distribution momentum widths for the dissociation of ${ }^{14} \mathrm{Be}$ at $35 \mathrm{MeV} /$ nucleon.

\begin{tabular}{ccccc}
\hline \hline & $\sigma_{-2 n}[\mathrm{~b}]$ & $\sigma_{n}[\mathrm{~b}]$ & $\bar{m}_{n}$ & $\Gamma_{n}[\mathrm{MeV} / c]$ \\
\hline $\mathrm{C}$ & $0.46 \pm 0.04$ & $0.75 \pm 0.10$ & $1.6 \pm 0.3$ & $75 \pm 3$ \\
$\mathrm{~Pb}$ & $2.3 \pm 0.4$ & $4.0 \pm 0.3$ & $1.7 \pm 0.2$ & $77 \pm 4$ \\
$\mathrm{~Pb}($ EMD) & $1.45 \pm 0.40$ & $2.7 \pm 0.4$ & $1.9 \pm 0.6$ & $87 \pm 6$ \\
\hline \hline
\end{tabular}


near threshold (see below) coupled with the weighting of the virtual photon spectrum to low photon energies [25].

Assuming that the neutron angular distribution arising from nuclear dissociation on $\mathrm{Pb}$ is identical to that measured for the $\mathrm{C}$ target, the single-neutron angular distribution for EMD has been constructed (Fig. 1b) and the corresponding integrated cross section and average multiplicity derived (Table I). Interestingly, the angular distribution remains narrow and the multiplicity is consistent with the value of 2 expected for EMD.

The invariant mass spectra, reconstructed from the measured momenta of the beam and fragments $\left({ }^{12} \mathrm{Be}\right.$ and two neutrons) from breakup, are displayed in Figs. 2a and $2 \mathrm{~b}$ for the $\mathrm{C}$ and $\mathrm{Pb}$ targets. The EMD spectrum (Fig. 2c) has been deduced, as described above, following subtraction of the estimated nuclear contribution to reactions on $\mathrm{Pb}$. As for the spectra obtained with the $\mathrm{C}$ and
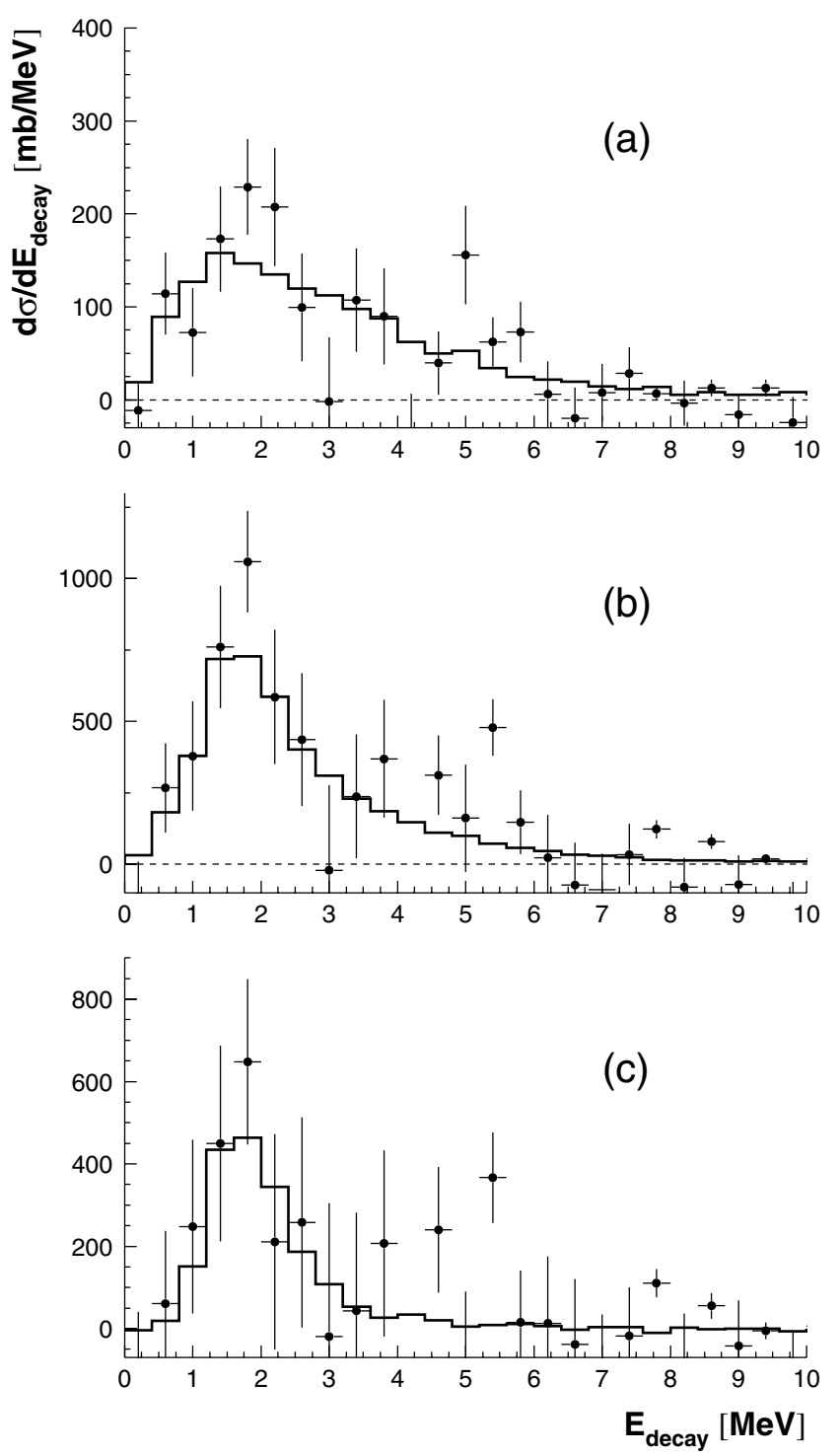

FIG. 2. Reconstructed ${ }^{14} \mathrm{Be}$ decay energy spectra for dissociation on (a) $\mathrm{C}$, (b) $\mathrm{Pb}$, and (c) that deduced for EMD on $\mathrm{Pb}$. The histograms correspond to the results of simulations (see text).
$\mathrm{Pb}$ targets, the EMD spectrum exhibits enhanced strength around $1.5 \mathrm{MeV}$ decay energy $\left(E_{\text {decay }}\right)$. Given the complex nature of the response function of the present setup, a detailed Monte Carlo simulation, including the influence of all nonactive materials, was developed based on the GEANT package [21]. The overall efficiency, assuming three-body phase-space decay, was constant $(\sim 0.5 \%)$ up to around $4 \mathrm{MeV}$ decay energy and decreased slowly with increasing energy $(\sim 0.05 \%$ at $10 \mathrm{MeV})$. The resolution in the reconstructed decay energy varied as approximately $\mathrm{FWHM}=0.54 E_{\text {decay }}^{0.5} \mathrm{MeV}$.

The results shown in Fig. 2 were obtained following the descriptions for dissociation on $\mathrm{C}$ and $\mathrm{Pb}$ outlined earlier and after filtering through the simulation. In the case of the nuclear induced reactions a single low-lying state in ${ }^{13} \mathrm{Be}\left(E_{0}=0.5 \mathrm{MeV}, \Gamma_{0}=0.5 \mathrm{MeV}\right)$ was assumed to be populated following the diffraction of one of the halo neutrons $[21,26]$. The EMD was simulated under the assumption that the energy sharing between the ${ }^{12} \mathrm{Be}$ and the two neutrons was governed by three-body phase space. As shown in Fig. 2c, the observed EMD decay energy spectrum could be reproduced assuming a Breit-Wigner line shape with $E_{0}=1.8 \pm 0.1 \mathrm{MeV}$ and $\Gamma_{0}=0.8 \pm$ $0.4 \mathrm{MeV}$. Furthermore, the corresponding simulations of the single-neutron angular distributions were in good agreement with those observed for reactions on $\mathrm{C}$ and $\mathrm{Pb}$, as well as that deduced for EMD [21].

As noted above, the EMD of halo nuclei is essentially $E 1$ in character. In the case of other well established halo nuclei, such as ${ }^{11} \mathrm{Be}$ and ${ }^{11} \mathrm{Li}$, the large $s$-wave components in the halo neutron configurations are reflected in high EMD cross sections and enhanced $E 1$ strength distributions near threshold $[1,27,28]$. The present observations would thus suggest that a significant $\nu\left(2 s_{1 / 2}\right)^{2}$ admixture is present in ${ }^{14} \mathrm{Be}$.

Thompson and Zhukov have examined ${ }^{14} \mathrm{Be}$ within the framework of a three-body model in which the ${ }^{12} \mathrm{Be}$ core is treated as inert [6] and a number of trial wave functions developed. Based on the binding energy and matter radius $[13,14]$ of ${ }^{14} \mathrm{Be}$, together with the known $d$-wave resonance at $2.01 \mathrm{MeV}$ in ${ }^{13} \mathrm{Be}$ [29], two ${ }^{14} \mathrm{Be}$ wave functions are favored (both of which require an $s$-wave state near threshold in ${ }^{13} \mathrm{Be}$ as suggested by recent experiments [26,30,31]): the so-called $D 4$ wave function $-86 \%$ $\nu\left(2 s_{1 / 2}\right)^{2}$ and $10 \% \nu\left(1 d_{5 / 2}\right)^{2}$; and $C 7-29 \% \nu\left(2 s_{1 / 2}\right)^{2}$ and $67 \% \nu\left(1 d_{5 / 2}\right)^{2}$. The EMD decay energy spectra calculated from the corresponding $E 1$ strength functions [6] for breakup at $35 \mathrm{MeV} /$ nucleon on $\mathrm{Pb}$ are compared in Fig. 3 with that of the empirical Breit-Wigner line shape deduced from the present measurements. The corresponding integrated two-neutron removal cross sections are $1.05 \mathrm{~b}(D 4)$ and $0.395 \mathrm{~b}(C 7)$ [6], compared to the measured value of $1.45 \pm 0.40 \mathrm{~b}$. Although the strength is predicted to be concentrated at a somewhat lower energy than that observed, a large $\nu\left(2 s_{1 / 2}\right)^{2}$ admixture to the valence neutrons wave function is favored. This conclusion is supported by the total reaction cross section 


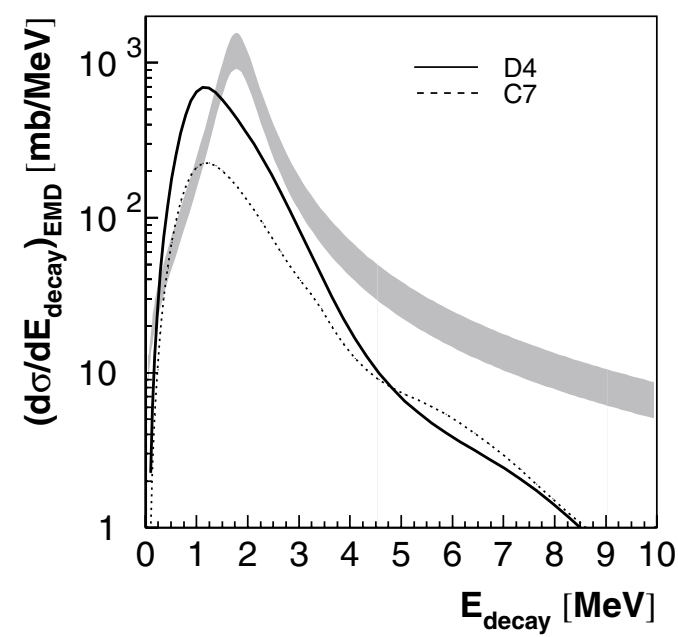

FIG. 3. Comparison of the EMD decay energy spectra for the three-body wave functions $D 4$ and $C 7$ [6] with that deduced from the present experiment (shaded band). The latter has been normalized to an integrated cross section of $1.45 \pm 0.40 \mathrm{~b}$.

measurement of Suzuki et al. [14] and is in line with the recent observation of a significant $\nu\left(2 s_{1 / 2}\right)^{2}$ component in the ground state of the neighboring $N=10$ isotone ${ }^{15} \mathrm{~B}$ [32]. Theoretically, such a configuration is also predicted by Lagrange mesh calculations [5,7]. It should be noted that the treatment of the core as inert precludes, $a b$ initio, the existence of any simple negative parity resonances in ${ }^{14} \mathrm{Be}$.

Descouvemont has explored ${ }^{13,14} \mathrm{Be}$ using a microscopic cluster model in which the core is active within the $p$ shell [33]. In the case of ${ }^{13} \mathrm{Be}$ an $s$-wave state is predicted very close to threshold, while the energy of the $d$-wave resonance is well reproduced. Significantly, a strong $E 1$ transition $\left[\mathrm{B}(E 1) \approx 1.2 e^{2} \mathrm{fm}^{2}\right]$ centered at $E_{\text {decay }}=1.5 \mathrm{MeV}$ is predicted in ${ }^{14} \mathrm{Be}$, very close to the structure observed experimentally. Analysis of the corresponding energy surface suggests, however, that this transition is not associated with a true resonance [33]. Calculations of the form of the associated continuum energy spectrum would be of considerable interest.

In conclusion, the first kinematically complete breakup reaction study of ${ }^{14} \mathrm{Be}$ has been reported. The twoneutron removal cross sections, neutron angular distributions, and invariant mass spectra for reactions on $\mathrm{C}$ and $\mathrm{Pb}$ were measured and the EMD contributions deduced. Comparison with model calculations suggest that the configuration of the halo neutrons contains a large $\nu\left(2 s_{1 / 2}\right)^{2}$ admixture. The enhanced strength observed near threshold in the EMD invariant mass spectrum is consistent with a nonresonant soft-dipole excitation. In terms of future work, exploration of higher energy continuum excitations would be of particular interest. Additionally, spectroscopic studies of ${ }^{13} \mathrm{Be}$ and the $\beta_{2}$ of ${ }^{12} \mathrm{Be}[34]$ are essential to developing more refined models of ${ }^{14} \mathrm{Be}$. In light of the breakdown in the neutron $p$-shell closure in ${ }^{12} \mathrm{Be}$ [35], the importance of $p$-sd cross shell excitations in the ${ }^{14} \mathrm{Be}$ ground state also merit exploration.
The authors are grateful to the support provided by the technical and operations staff of LPC and GANIL. Discussions with Ian Thompson and Pierre Descouvemont are also acknowledged.

*Present address: Department of Electronic Engineering and Physics, University of Paisley, Paisley, United Kingdom.

†Electronic address: ORR@CAELAV.IN2P3.FR

†Present address: ISOLDE, CERN, Genève, Switzerland.

${ }^{\S}$ On leave from Institut de Physique Nucléaire, UCL, Louvain-la-Neuve, Belgium.

"Present address: Department of Physics, University of Edinburgh, Edinburgh, United Kingdom.

[1] P. G. Hansen et al., Annu. Rev. Nucl. Part. Sci. 45, 591 (1995).

[2] P. G. Hansen and B. Jonson, Europhys. Lett. 4, 409 (1987).

[3] K. Riisager et al., Nucl. Phys. A548, 393 (1992).

[4] Z. Ren et al., Phys. Lett. B 351, 11 (1995).

[5] A. Adahchour et al., Phys. Lett. B 356, 445 (1995).

[6] I. J. Thompson and M. V. Zhukov, Phys. Rev. C 53, 708 (1996); (private communication).

[7] D. Baye, Nucl. Phys. A627, 305 (1997).

[8] K. Riisager et al., Nucl. Phys. A540, 365 (1992).

[9] M. Zahar et al., Phys. Rev. C 48, R1484 (1993).

[10] M. Zahar et al., Phys. Rev. C 49, 1540 (1994).

[11] I. Tanihata et al., Phys. Lett. B 206, 592 (1988).

[12] E. Liatard et al., Europhys. Lett. 13, 401 (1990).

[13] J. S. Al-Khalili et al., Phys. Rev. C 54, 1843 (1996).

[14] T. Suzuki et al., Nucl. Phys. A658, 313 (1999).

[15] R. Gilman et al., Phys. Rev. C 30, 958 (1984).

[16] J. M. Wouters et al., Z. Phys. A 331, 229 (1988).

[17] K. Ikeda, Nucl. Phys. A538, 355c (1992).

[18] T. Kobayashi et al., Phys. Lett. B 232, 51 (1989).

[19] F. M. Marqués et al., Phys. Lett. B 476, 219 (2000).

[20] F. M. Marqués et al., Nucl. Instrum. Methods Phys. Res., Sect. A 450, 109 (2000).

[21] M. Labiche, Thèse, Université de Caen [LPCC T-99-03 (to be published)].

[22] F. Barranco et al., Phys. Lett. B 319, 387 (1993).

[23] F. Barranco et al., Z. Phys. A 356, 45 (1996).

[24] C. A. Bertulani and A. Sustich, Phys. Rev. C 46, 2340 (1992).

[25] M.S. Hussein et al., Phys. Rev. C 44, 2219 (1991).

[26] K. L. Jones, Thesis, University of Surrey (to be published).

[27] T. Nakamura et al., Phys. Lett. B 331, 296 (1994).

[28] M. Zinser et al., Nucl. Phys. A619, 151 (1997).

[29] A. N. Ostrowski et al., Z. Phys. A 343, 489 (1992).

[30] M. Thoennessen, in Proceedings of the International School of Heavy-Ion Physics, 4th Course: Exotic Nuclei, edited by R. A. Broglia and P. G. Hansen (World Scientific, Singapore, 1998), p. 269.

[31] M. Belozyorov et al., Nucl. Phys. A636, 419 (1998).

[32] E. Sauvan et al., Phys. Lett. B 491, 1 (2000); E. Sauvan, Thèse, Université de Caen [LPCC T-00-01 (to be published)].

[33] P. Descouvemont, Phys. Rev. C 52, 704 (1995); (private communication).

[34] H. Iwasaki et al., Phys. Lett. B 481, 7 (2000).

[35] A. Navin et al., Phys. Rev. Lett. 85, 266 (2000), and references therein. 